\title{
A MÚSICA NA CIÊNCIA DA INFOMAÇÃO: uma análise de domínio da temática no Brasil no período de 1972 a 2018
}

Emanuella Maria Barbosa Lourenço Bezerra ${ }^{1}$, Andrea Carla Melo Marinho ${ }^{2}$, Francisco Arrais Nascimento $^{3}$, Daniel Martínez-Ávila ${ }^{4}$, Fabio Assis Pinho ${ }^{5}$

1 orcid.org/0000-0002-4886-7557, Comissão Memorial na Universidade de Pernambuco UPE/Pronto-Socorro Cardiológico de Pernambuco Prof. Luiz Tavares - PROCAPE/Biblioteca Prof. Ovídio Montenegro - BPOM, Recife, Pernambuco, E-mail: emanuella.bezerra@upe.br

2 orcid.org/0000-0001-6957-6911, Programa de Pós-graduação em Comunicação da Universidade Federal do Rio Grande do Sul, Porto Alegre, Rio Grande do Sul, Brasil. E-mail: andreacmmarinho@gmail.com

3 orcid.org/0000-0003-4424-8844, Programa de Pós-graduação em Ciência da Informação da Universidade Estadual Paulista Júlio de Mesquita Filho, Marília, São Paulo, Brasil. E-mail: francisco.arrais.nascimento@gmail.com

4 orcid.org/0000-0003-2236-553X, Departamento de Ciência da Informação da Universidade Estadual Paulista Júlio de Mesquita Filho, Marília, São Paulo, Brasil. E-mail: martinez.avila@unesp.br

5 orcid.org/0000-0003-1346-3808, Universidade Federal de Pernambuco, Recife, Pernambuco, Brasil. E-mail: fabiopinho@ufpe.br

Tipo de trabalho: comunicação

Palavras-chave: Música; Ciência da Informação; Análise de Domínio; Organização da Informação.

\section{Introdução}

O estudo identifica como o objeto "música", vem auferindo espaço em pesquisas interdisciplinares, permitindo a visualização de tal objeto enquanto domínio na Ciência da Informação (CI). Nesse sentido, a CI sob o viés sociocultural tem ampliado seu escopo de pesquisas, trabalhando a informação a partir dos domínios diferenciados. Assim, objetos não convencionais como os artefatos culturais, configuram-se como fonte de informação e consequentemente objeto de estudo na área. Barros, Café e Almeida (2013), postulam que a "materialidade sonora da música", não pode ser trabalhada apenas sob a perspectiva de sua sonoridade, mas sim, atentar para o seu conteúdo. Logo, a música fora eleita nesta pesquisa por sua universalidade e pela afinidade dos autores com o objeto e sob a égide da CI, dada sua natureza interdisciplinar, bem como por interseccionar arte e ciência.

Para compreender a atuação de tal objeto na CI, não ignorando sua relação e sentidos social e histórico, alocando a canção enquanto recurso informacional, indagou-se: Quantos trabalhos em CI contemplam a música como objeto de pesquisa no Brasil? Quais as perspectivas de análise destas pesquisas? Quais os meios de comunicação científica que os trabalhos foram divulgados?

Para tal empreendimento, foram mapeadas as pesquisas desenvolvidas nos programas de pósgraduação em Ciência da Informação, Gestão da Informação, Biblioteconomia, Comunicação e Informação e Memória Social, com dados atualizados do relatório de área de 2017 da Coordenação de Aperfeiçoamento de Pessoal de Nível Superior (CAPES), além dos artigos de periódicos e de anais 
de eventos publicados nas principais bases de dados da área que contemplassem a música enquanto objeto de pesquisa em Ciência da Informação no Brasil.

Assim, o trabalho tem por objetivo analisar a produção científica brasileira sobre música no âmbito da Ciência da Informação.

\section{Música e Ciência da Informação}

A música, como parte da cultura ocidental está intimamente ligada à cultura medieval, visto que muito das memórias deste período foram pautadas por meio da tradição oral e com formas de consumo e produção fechados, circunscrita a pequenos grupos ou aldeias (IAZZETTA, 2001). Com o surgimento da imprensa, houve a possibilidade de novas formas de difusão do conhecimento e da informação, permitindo materializar a informação sonora da música, bem como sua disseminação, mas é só por meio do fonógrafo que foi possível alavancar sua difusão. Assim, o registro informacional da música (partitura e letra da canção) possibilitou seu registro como fonte documental de memória.

Le Coadic (2004, p. 5) afirma que a informação “[...] é um conhecimento inscrito" em qualquer suporte. Otlet (2018) faz alusão sobre a "documentação sonora", onde a música e outros documentos sonoros estão aí contemplados, visto que para o autor tanto do ponto de vista da música quanto das letras, "o disco" é um tipo de documentação. Briet (2016), lança mão de um exemplo clássico quando pergunta se um "antílope é um documento?" e responde que não, mas tudo o que for produzido a partir dele é um documento. Da mesma forma, o som produzido por um instrumento não é um documento, mas a sua composição, as letras produzidas, os discos, os Compact Disc (CDs), entre outros são documentos.

Esse raciocínio evidencia não apenas a vertente informacional da música, mas as possibilidades de estudos utilizando-se deste objeto. Morigi e Bonotto (2004) apontam para o tratamento da música nos campos da Arquivologia, Biblioteconomia e Museologia, que tais objetos são tratados de forma a respeitar as várias facetas que se apresentam e não apenas às formas tradicionais no formato em papel. Nesse sentido, Toller e Bizello (2017, p. 272) colocam que o "[...] documento tem seu valor definido aos olhos do pesquisador" visto que tal registro é importante, independentemente da forma como ele se apresente.

Saracevic (1996) evidencia que a CI apresenta três características que constituem a razão de sua existência e evolução, ou seja, sua natureza interdisciplinar, sua relação com a tecnologia da informação e sua contribuição na evolução da sociedade da informação, uma vez que apresenta uma forte dimensão social e humana.

Nesse sentido, é possível considerar que os estudos acerca de artefatos artístico-culturais enquanto objeto de pesquisa em CI apresentam as características mencionadas anteriormente, uma vez que há uma forte relação com outros campos de conhecimento (artes, ciências sociais, cultura e linguística), bem como essa produção é armazenada em unidades de informação de instituições acadêmicas e culturais, ou mesmo em ambientes digitais.

Para além de tal compreensão, Von Simson (2006, p. 1), associa a música às questões memoriais, tratando-a não apenas como artefato informacional e cultural, mas sim, um documento imagético (impregnada de imagens, lembranças, memórias), além de meio de representações sociais, evidenciando a capacidade do ser humano de guardar fatos e experiências, bem como a necessidade não só da guarda, mas da difusão destes fatos para a posteridade, utilizando-se para isso, além da história oral, variados suportes de informação, tais como mecanismos de voz, imagem e texto. Em consonância com o autor, Morigi \& Bonotto (2004, p. 148) falam sobre a narrativa musical que objetiva "expressar os sentimentos coletivos" e que tais documentos estão impregnados de sentido, que vai para a além da racionalidade, mas carrega em si muito do que retém os sentimentos. 
Susana Sardo (2018, pp.9-10) afirma que "[...] o primeiro desafio que se nos depara quanto tentamos escrever ou falar sobre música é o de não saber como nomeá-la. [...] Por tanto, o exercício de objetificação, de fixação e de classificação da música é complexo e começa, desde logo, no momento de sua nomeação". Estas reflexões tem relação com a tradição crítica da CI e a Organização do Conhecimento e as consequências éticas do "poder de nomear" (OLSON, 2002).

Percebe-se que a temática favorece uma discussão acerca do campo científico da CI, uma vez que possibilita uma diferente perspectiva de análise do seu objeto de estudo. De acordo com Santos \& Kobashi (2007, p.106), quando falam daquilo que permeia e abaliza o campo da CI, visto que seus objetos de estudo estão ligados aos fatores "[...] epistemológicos, teóricos e metodológicos das disciplinas científicas", tendo entre seus pares o entendimento necessário sobre os princípios, autenticidade, adequação das discussões propostas e de sua razoabilidade e pertinência das metodologias, procedimentos e ferramentas próprias para o desenvolvimento de pesquisas.

\section{Aspectos Metodológicos}

A pesquisa de natureza descritiva, arraigada sob análise de domínio, ancorando-se nas contribuições de Tennis (2002, 2003, 2012), Hjørland (1997, 2002, 2003, 2017) e Hjørland \& Albrechtsen (1995). Apresenta como corpus 124 produções acadêmicas (artigos de periódicos, artigos provenientes de anais de eventos, dissertações e teses), que utilizam a música como objeto de pesquisa, publicados no período de 1974 a 2018, identificados nas seguintes fontes: Base de Dados Referencial de Artigos e Repositórios em Ciência da Informação (BRAPCI), Base de dados dos Anais dos Enancib (BENANCIB), Anais do Capítulo Brasil da International Society for Knowledge Organization (ISKO), Base de dados Rede PERI, da UFMG e na Biblioteca Digital Brasileira de Teses e Dissertações (BDTD) do IBICT, Banco de Teses da CAPES e Repositórios Institucionais aos quais estão vinculados os 30 Programas de Pós-Graduação em Ciência da Informação no Brasil.

Os termos de busca utilizados foram "Música" e "Musical", e após recuperação, os trabalhos foram organizados de acordo com uma categorização do conteúdo referente à temática da produção analisada. Assim, foram definidas 4 categorias de análise de acordo com a Figura 1.

Figura 1: Categorias de Análise

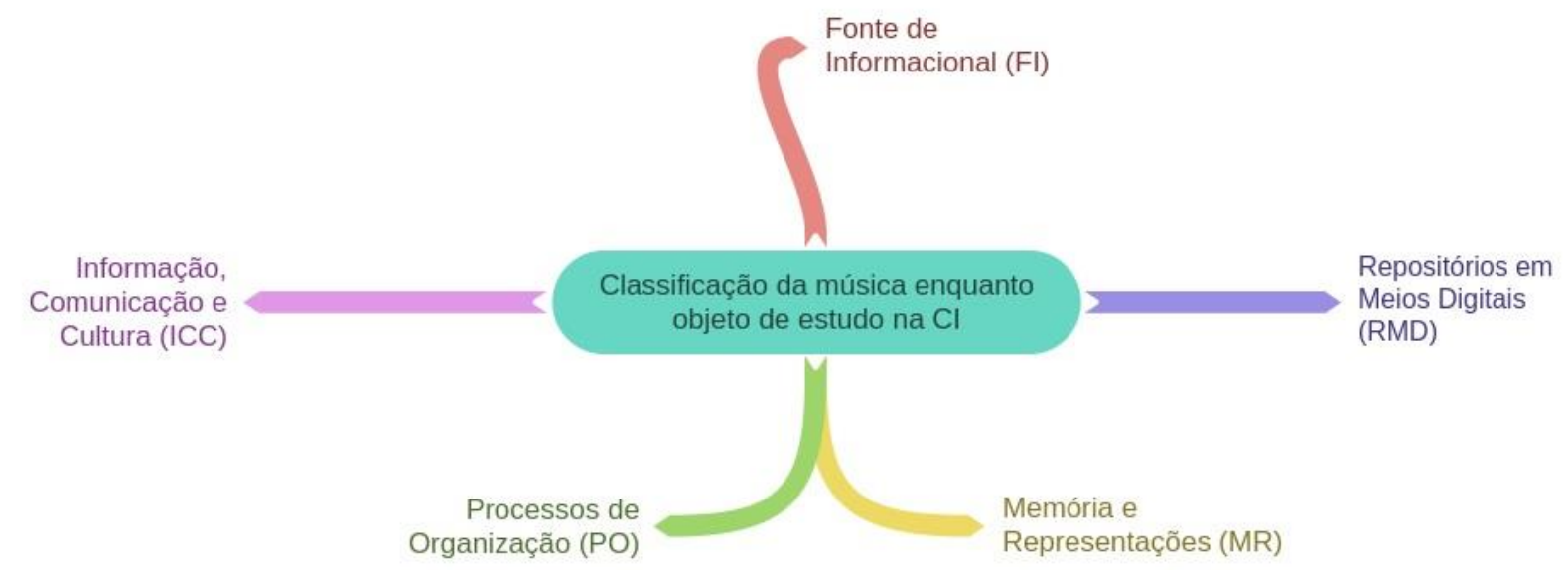

\section{Análise e Discussão Dos Resultados}

Na BRAPCI foram selecionados 37 artigos produzidos no período de 1974 a 2018. Na base PERI foram recuperados 29 registros, publicados no período de 1980 a 2016. Na BENANCIB foram 
encontrados 33 artigos, publicados entre 1995 e 2018. Vale ressaltar que houve duplicidade na indexação de trabalhos nas bases PERI, BRAPCI e BENANCIB. Assim, foram encontrados 2 trabalhos iguais na PERI e BENANCIB e 11 entre a PERI e BRAPCI. Quanto aos eventos, nos Anais da ISKO Brasil foram encontrados 4 trabalhos nas edições do evento que foram realizadas nos anos 2013-2017.

Figura 2: Produção na Área de Ciência da Informação segundo a Fonte e a Classificação Estabelecida pelos autores

\begin{tabular}{|c|c|c|c|c|c|}
\hline \multicolumn{6}{|l|}{$100 \%$} \\
\hline \multicolumn{6}{|l|}{$80 \%$} \\
\hline \multicolumn{6}{|l|}{$60 \%$} \\
\hline \multicolumn{6}{|l|}{$40 \%$} \\
\hline \multicolumn{6}{|l|}{$20 \%$} \\
\hline \multirow{2}{*}{$0 \%$} & FI & ICC & MRS & PO & RMD \\
\hline & FI & ICC & MRS & PO & RMD \\
\hline$\square$ PERI & 4 & 0 & 4 & 10 & 3 \\
\hline 口ISKO Brasil & 2 & 0 & 1 & 4 & 1 \\
\hline$\square$ BRAPCI & 27 & 4 & 1 & 17 & 11 \\
\hline$\square$ BENANCIB & 11 & 8 & 8 & 21 & 7 \\
\hline
\end{tabular}

A figura 2 demonstra a concentração e produtividade temática com o objeto música por meio da coleta dos artigos nas bases em CI no Brasil. Os resultados apontam que a pesquisa acerca do objeto tem crescido nos últimos anos, tendo a área trabalhado o objeto na maior parte dos trabalhos sob um viés documental, porém outras perspectivas de estudo com o objeto, a exemplo da música enquanto fonte de memória e representação social, o acesso e preservação de recursos audiovisuais em ambientes digitais também podem ser visualizadas.

Por fim, foi analisada a produção de dissertações e teses no Brasil, onde buscou-se na Banco de Teses da CAPES, BDTD, e nos repositórios das instituições (UNB, UFBA, UFPB, UFSC e USP) para fins de comprovação, totalizando 24 trabalhos (16 dissertações e 8 teses) (Figura 3). Sobre a temática da música em 8 programas de pós-graduação como é possível observar na Figura 4.

Figura 3: Produção cientifica segundo o nível de graduação

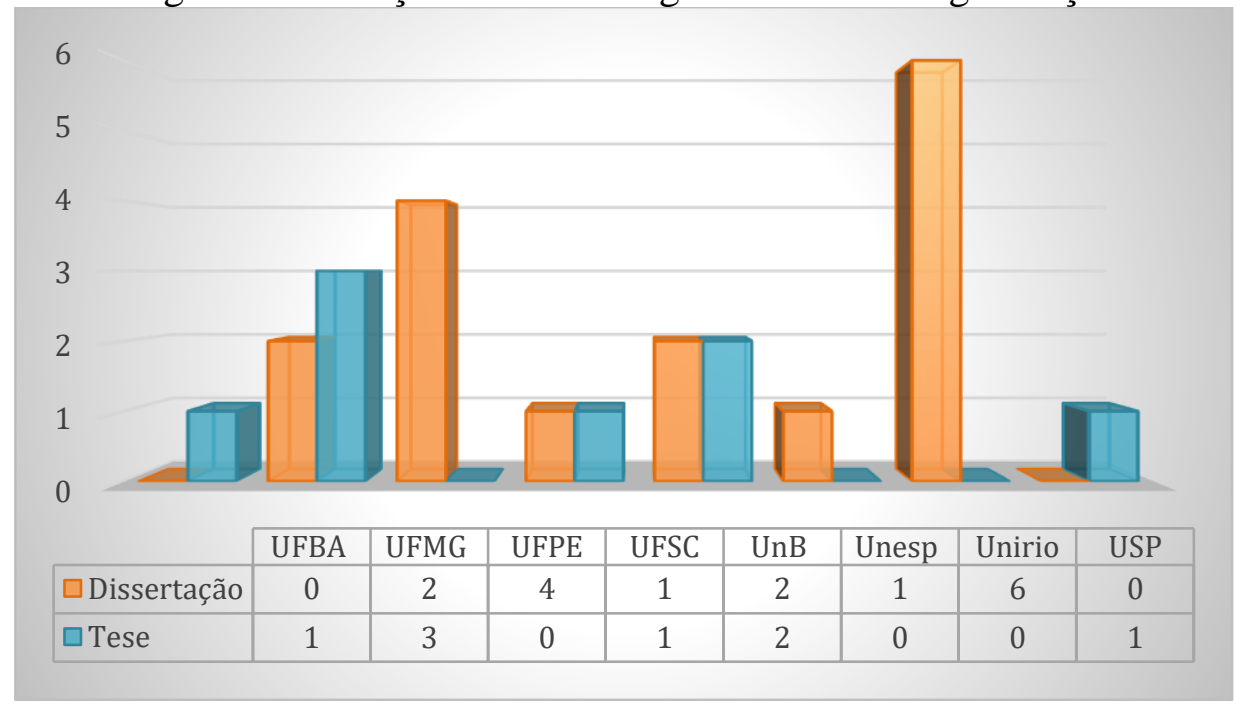


A figura 3 demonstra a distribuição de trabalhos acadêmicos produzidos nos 8 PPG's em CI no Brasil segundo a classificação estabelecida. Observou-se uma concentração de pesquisas desenvolvidas predominantemente no programa de Memória Social da UNIRIO com 6 dissertações. Contribuindo para o aumento da produtividade no domínio seguem o PPGCI-UFMG com 2 teses e 3 dissertações, o PPGCI-UNB com 2 teses e 2 dissertações, o PPGCI-UFPE com 4 dissertações e o PPGCI-UFSC com 1 tese e 1 dissertação. Os programas da UFBA, UNESP-Marília e USP produziram 1 dissertação.

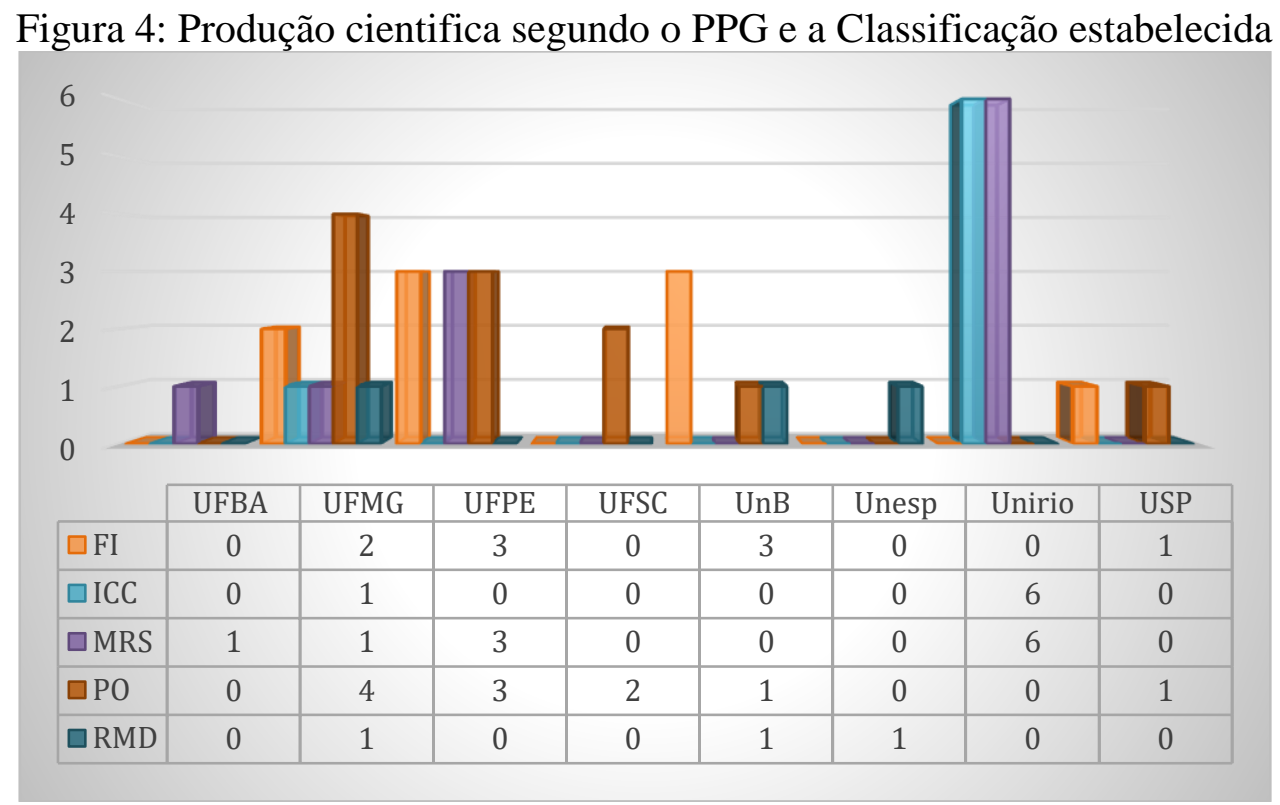

A Figura 4 mostra que o objeto é discutido proporcionalmente nas diferentes categorias de análise, o que difere dos dados referentes às bases em CI e aos anais do ENANCIB. Percebe-se que os estudos acerca da música, que se configura enquanto manifestação artístico-cultural são crescentes na CI e possibilitam uma discussão teórico-metodológica no âmbito da OI e estudos de memória, sobretudo a partir de produções do programa em Memória Social da Unirio, que concentra a maior produção de pesquisas sobre a temática, seguido dos PPGs da UFMG, UnB, UFPE e UFSC.

\section{Considerações Finais}

Observou-se que a categoria de análise predominante é a que trata a música sob um viés documental, voltado a estudos de organização de acervos musicais no que tange aos processos de catalogação, classificação e indexação desses recursos informacionais. Porém, observa-se a emergência de outras perspectivas de análise em trabalhos que têm direcionado suas pesquisas sobre aspectos socioculturais acerca do objeto, sobretudo no que se refere à música enquanto recurso informacional de memória e representação social.

\section{Referências}

Barros, C. M. de; Café, L. M. A. \& Almeida, C. C. de. (2013). Informação musical e interpretação: contribuições semióticas para o campo da organização da informação. In: Anais, 14 Encontro Nacional de Pesquisa em Ciência da Informação, [online] São Luís: UFMA. Recuperado a partir de: 
http://200.20.0.78/repositorios/bitstream/handle/123456789/2335/INFORMA\%C3\%87\%C3\%83O \%20MUSICAL.pdf?sequence=1. Acesso em: $01 \mathrm{dez} .2018$.

Briet, S. (2016). O que é a Documentação? [Ebook]. Brasília: Briquet de Lemos. Recuperado a partir de: http://file:///C:/Users/AcerOficial/Downloads/Briet_Suzanne_o_que_eha_documenta\%C3\%A7\%C3\%A3o.pdf.pdf

García Gutiérrez, A. (2018). En Pedazos: el sentido de la desclasificación. Madrid: ACCI.

Hjørland, B. (2017). Domain analysis. Knowledge Organization, 44(6), 436-464.

Hjørland, B. (2002). Domain analysis in information science. Eleven approaches - traditional as well as innovative. Journal of Documentation, 58(4), 422-462.

Hjørland, B. (2003). Fundamentals of knowledge organization. Knowledge Organization, 30(2), 87111.

Hjørland, B. (1997). Information seeking and subject representation: an activity theoretical approach to Information Science. London: Greenwood Press.

Hjørland, B. \& Hanne A. (1995). Toward a new horizon in information science: Domain analysis. Journal of the American Society for Information Science, 46(6), 400-425.

Iazzetta, F. (2001). Reflexões sobre música e o meio. In: Anais, 13 Encontro da Associação Nacional de Pesquisa e Pós-Graduação em Música [online] Belo Horizonte, UFMG: Recuperado a partir de:

http://www.anppom.com.br/anais/anppom_2001_1.pdf. Acesso em: 10 dez. 2018.

Le Coadic, Y. (2004). A ciência da informação. 2. ed. Brasília: Briquet de Lemos.

Morigi, V., \& Bonotto, M. (2004). A Narrativa Musical, Memória e Fonte de Informação Afetiva. Em Questão, 10 (1), 143-161.

Olson, H. A. (2002). The power to name: locating the limits or subject representation in libraries. Dordrecht: Kluwer Academic Publisher.

Otlet, P. (2018). Tratado de documentação: o livro sobre o livro: teoria e prática. [Ebook]. Brasília: Briquet de Lemos. Retrieved from http://www.cfb.org.br/wpcontent/uploads/2018/09/otlet_tratado_de_documenta\%C3\%A7\%C3\%A3o.pronto.pdf

Santos, R. and Kobashi, N. (2007). Análise de teses e dissertações de Ciência da Informação: estudo de institucionalização de um campo científico. In: Anais, 8 Encontro Nacional de Pesquisa em Ciência da Informação. [online] Salvador: Universidade Federal da Bahia. Recuperado a partir de: http://www.enancib.ppgci.ufba.br/artigos/GT7--109.pdf [Accessed 1 Dec. 2018].

Saracevic, T. (1996). Ciência da informação: origem, evolução e relações. Perspectivas em Ciência da Informação, 1 (1), 41-62. 
Sardo, S. (2018). Presentación. O desconforto de escrever sobre música. In: García Gutiérrez, A. (2018). En Pedazos: el sentido de la desclasificación. Madrid: ACCI.

Tennis, J. (2012). A convenient verisimilitude or oppressive internalization? Characterizing the ethical arguments surrounding hierarchical structures in knowledge organization systems. Knowledge Organization, 39 (5), 394-397.

Tennis, J. T. (2002). Subject ontogeny: Subject access through time and the dimensionality of classification. In: López-Huertas, M. J. (Ed.). Challenges in knowledge representation and organization for the 21st Century: Integration of knowledge across boundaries: Proceedings of the Seventh International ISKO Conference, Granada (Spain), 2002. Würzburg: Ergon. 8, 54-59.

Tennis, J. T. (2003). Two axes of domains for domain analysis. Knowledge Organization, 30, nos. 3-4: 191-195.

Toller, M. E. and Bizello, M. L. (2017). As práticas documentais e a propagação da memória social. In: M. A. Alves, M. C. Grácio and D. Martinez-Ávila, ed., Informação, conhecimento e modelos, 1st ed. [online] Marília: Oficina Universitária, pp.271-284.

Von Simson, O. (2003). Memória, cultura e poder na sociedade do esquecimento. Revista Acadêmica, (6), 14-18. 